\title{
DENSITY OF SURGICAL SPECIMENS AND CORRELATION WITH CLINICAL CHARACTERISTICS OF WOMEN SUBMITTED TO MASTECTOMY: THE ARCHIMEDES PROJECT
}

Cassio Cardoso Filho1, Mariana Sousa Sguerra Silva, Ana Gabriela Bicalho Rabelo', Cesar Cabello dos Santos", Giuliano Mendes Duarte'

${ }^{1}$ Universidade Estadual de Campinas - Campinas (SP), Brazil.

Introduction: Breast cancer is the second most common neoplasm among women, being responsible, per year, for 60 thousand new cases of breast cancer in Brazil. The treatment varies according to staging, and mastectomy is indicated in cases in which conserving surgery it is not possible. Mastectomy patients who do not present with indication for breast surgical reconstruction may have self-esteem problems, and external prostheses are one of the means to recover body image. Despite being an external, relatively simple device, external prosthesis may lead do pruritus, pain, paresthesia and postural changes. Therefore, the objective of this study is to describe the density of the female breast to serve as a base to improve these prostheses, considering that the current literature lacks studies to establish this parameter. Objectives: To determine the density of the female breast and correlate it with the clinical and epidemiological characteristics and the treatment. Subjects and Methods: This is a descriptive study that measured the mass and volume of 100 mastectomy specimens to calculate density, with correlation to factors such as age, parity, menstrual factors. Determination of frequencies, means and standard-deviation of the variables, with risk assessment, was carried out with the chi-square or the Fisher's exact test for the expected values lower than 5, as well as the log-binomial model between the variables using the prevalence ratio with 95\% confidence interval (GraphPad, available at https://www.graphpad.com/quickcalcs/contingency $2 /$ ). We considered $\mathrm{p}<0.05$ as significant, and confidence intervals (CI) were established at $95 \%$. Results: Of the 100 women included in this study, 78\% were white, mean age was 58 years (300-94 years), mean age at menarche was 13 years, and mean age at menopause was 49 years. Of the women submitted to mastectomy, $39 \%$ were in their reproductive years. Mean density of the mastectomy specimens in this study was $1.23 \mathrm{~g} / \mathrm{cm}^{3}$. There was statistically significant correlation regarding the difference in the density of the specimens in patients with Body Mass Index (BMI) $>30$ in relation to the density of the specimens in patients with BMI $<30$. In this aspect, we found lower density associated with BMI $>30$ ( $p=0.0025)$. Conclusion: unlike what we found in the literature, the mastectomy specimens are approximately $20 \%$ denser in relation to water and silicone, which are commonly used materials in internal and external prosthesis. This reference can collaborate with the development of prosthesis that are more similar to the natural breast, better adjusting to the daily life of women, with fewer side effects and better quality of life. 